\title{
Long-Term High-Precision Monitoring from the Geneva Photometric Database
}

\author{
G. Burki, F. Kienzle \\ Geneva Observatory, CH-1290 Sauverny, Switzerland
}

\begin{abstract}
Very small amplitude variable stars are detected in the Geneva photometric database. Two examples of periodic variable stars with semi-amplitudes of 6.9 and $2.9 \mathrm{mmag}$ are presented.
\end{abstract}

\section{Introduction}

During the past 39 years, 345,000 measurements have been obtained in the Seven-Colour Geneva Photometric System on 48,000 stars (Rufener 1988; Burki et al. 1999; Cramer 1999). Six stations have been used: La Silla (Chile), Jungfraujoch and Gornergrat (Switzerland), Haute-Provence (France), Calar Alto and Isana (Spain). The accuracy of these photometric data varies from 3 to 7 mmag (Burki, Cramer, \& Nicolet 1991) and these measurements are homogeneous, i.e. they can all be inter-compared. A large number of new periodic very-small amplitude variable stars has been detected, in particular among our photometric standard stars, because these stars have been frequently measured, over a large time interval (e.g. Carrier, Burki, \& Richard 1999). Among the small amplitude variable stars, those which are periodic are much more easily detected. Two examples are presented here.

\section{New Extremely Small Amplitude Variable Stars}

The K4Ve-type star HR 8721 (see Figure 1a) is known as a BY Dra variable star. Busko \& Torres (1978) have proposed a period of $10.3 \mathrm{~d}$ and an amplitude of 0.04 mag. Our analysis, based on an independent detection of the variability of this star, shows that, at least in the period 1976-1982, the amplitude was much smaller, 6.9 mmag (semi-amplitude), and the period is precisely determined, $10.0173 \mathrm{~d}$.

The Be star HR 1960 (see Figure 1b) has been monitored from 1982 to 1998 in Geneva photometry and from November 1989 to March 1993 by the Hipparcos satellite. This star shows a periodic light variation with a period of $395 \mathrm{~d}$, characterised by an extremely small semi-amplitude, 2.9 millimags in $V$. This is most probably the long-period variable star with the smallest amplitude yet known. This variability is attributed to the Be characteristics of the star. A model similar to that of Carrier et al. (1999) for HR 2968 is proposed: the $\mathrm{Be}$ star could be the main component of a binary system having an orbit of period $395 \mathrm{~d}$. 

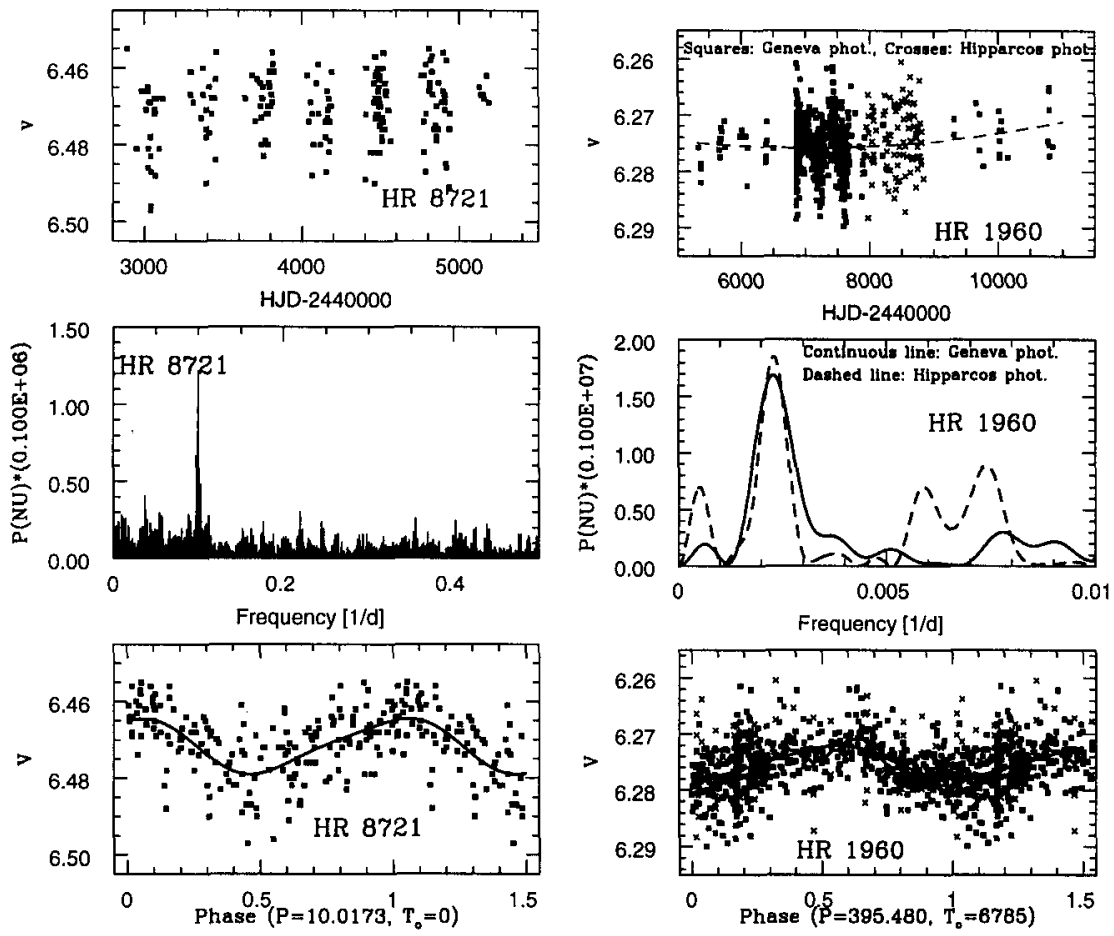

Figure 1. Extremely small amplitude periodic variable stars. a) HR 8721 a K4Ve-type star (6.9 mmag); b) HR 1960 a Be-type star (2.9 mmag).

\section{Conclusion}

Variable stars with amplitudes of a few mmag can be easily detected from accurate and homogeneous photometric data if they are periodic. Indeed, the fact that the variability is periodic offers a major possibility for the detection of very small amplitude oscillations, since the Fourier analysis may be used in that case. Such variable stars would not have been detected on the basis of their standard deviation exclusively.

\section{References}

Burki, G., Grenon, M., Richard, C., Pernier, B., Nicolet, B., \& Cramer, N. 1999, Geneva Photometric Database, http://obswww.unige.ch

Burki, G., Cramer, N., \& Nicolet, B. 1991, A\&AS, 87, 163

Busko, I. C. \& Torres, C. A. O. 1978, A\&A, 64, 153

Carrier, F., Burki, G., \& Richard, C. 1999, A\&A, 341, 469

Cramer, N. 1999, New Astronomy Reviews, in press

Rufener, F. 1988, Geneva Photometric Catalogue, $4^{\text {th }}$ ed., Obs. Genève 